\title{
Spatial resolution and switching field of magnetic force microscope tip coated with FePd-alloy thin film
}

\author{
Shinji Ishihara, Mitsuru Ohtake, and Masaaki Futamoto \\ Faculty of Science and Engineering, Chuo University, 1-13-27 Kasuga, Bunkyo-ku, Tokyo, 112-8551, Japan
}

\begin{abstract}
Magnetic force microscope (MFM) tips are prepared by coating Si tips of $4 \mathrm{~nm}$ radius with $L 1_{0}$ ordered FePd-alloy films varying the thickness in a range between 10 and $80 \mathrm{~nm}$. The effects of coating thickness on spatial resolution and switching field of MFM tip are investigated. As the thickness increases from 10 to $20 \mathrm{~nm}$, the MFM signal detection sensitivity is improved and the resolution improves from 12.7 to $7.9 \mathrm{~nm}$. With further increasing the thickness, the resolution decreases due to increase of tip radius. Magnetic bits of $15.9 \mathrm{~nm}$ length of a perpendicular medium recorded at 1600 kilo-flux-change-per-inch are distinguishable in the MFM image observed by using a tip coated with 20-nm-thick FePd film. The switching field monotonically increases from 0.70 to $1.50 \mathrm{kOe}$ with increasing the coating thickness from 10 to $80 \mathrm{~nm}$. The present study has shown that it is possible to prepare an MFM tip with spatial resolution better than $10 \mathrm{~nm}$ and switching field higher than $1 \mathrm{kOe}$ by coating a sharp Si tip with an $L 1_{0}$ ordered FePd-alloy film.
\end{abstract}

\section{Introduction}

Magnetic force microscopy (MFM) has been widely used to investigate the magnetization structures of magnetic devices like hard-disk-drive (HDD) media, permanent magnets, etc. MFM tips are generally prepared by coating non-magnetic sharp tips with magnetic materials [1]-[7]. The tip shape and the magnetic property of coated material influence the spatial resolution and the switching field $\left(H_{\mathrm{sw}}\right)$ of MFM tip.

The areal density of HDD medium is now approaching to $1 \mathrm{~Tb} / \mathrm{in}^{2}$ and expected to further increase. Future HDD media are considered to consist of magnetic materials with very high uniaxial magnetocrystalline anisotropy energies such as $\mathrm{FePt}$ and $\mathrm{SmCo}_{5}$. Therefore, realization of high $H_{\mathrm{sw}}$ is required in addition to highresolution better than $10 \mathrm{~nm}$ for MFM observations of such ultra-high-density media. MFM tips with high $H_{\text {sw }}$ have been prepared by annealing tips coated with $L 1_{0}$ ordered FePt- and CoPt-alloy films followed by focusedion-beam milling [6, 7]. However, the resolution of commercially available MFM tip coated with $L 1_{0}$ ordered film is limited at around $20 \mathrm{~nm}$.

In order to improve the resolution, it is necessary to reduce the magnetic volume interacting with a magnetic sample. In our previous studies [8]-[10], MFM tips were prepared by coating Si tips with magnetic materials such as $\mathrm{Co}-\mathrm{Cr}-\mathrm{Pt}, \mathrm{Co}, \mathrm{Fe}, \mathrm{Ni}, \mathrm{Ni}-\mathrm{Fe}$, and Fe-B. Spatial resolutions around $10 \mathrm{~nm}$ were obtained by adjusting the coating film thickness. An FePd-alloy film is reported to show a higher degree of $L 1_{0}$ ordering than FePt- and
CoPt-alloy films, when prepared by using a similar hightemperature process [11]. In the present study, MFM tips are prepared by coating Si tips with FePd films. The effects of coating thickness on resolution and $H_{\mathrm{sw}}$ are investigated.

\section{Experimental procedure}

MFM tips were prepared by coating base-Si tips of $4 \mathrm{~nm}$ radius with thin films by employing a radio-frequency (RF) magnetron sputtering system with base pressures lower than $4 \times 10^{-7} \mathrm{~Pa}$. $\mathrm{Fe}_{50} \mathrm{Pd}_{50}$ (at. \%) and $\mathrm{MgO}$ targets of 3 in diameter were used. The distance between target and Si tip was $150 \mathrm{~mm}$. The Ar gas pressure was kept constant at $0.67 \mathrm{~Pa}$. The RF powers for FePd and $\mathrm{MgO}$ targets were fixed at 35 and $200 \mathrm{~W}$, where the deposition rates were 0.020 and $0.015 \mathrm{~nm} / \mathrm{s}$, respectively. $\mathrm{MgO}$ underlayers and $\mathrm{FePd}$ films were sequentially formed on base-Si tips at room temperature (RT). The $\mathrm{MgO}$ underlayer was introduced to avoid atomic mixing between the Si tip and the FePd film. The thickness of $\mathrm{MgO}$ underlayer was fixed at $5 \mathrm{~nm}$, while that of $\mathrm{FePd}$ film was varied in a range between 10 and $80 \mathrm{~nm}$. The thicknesses were estimated for films deposited on flat substrates which were located near the base-Si tips. After film formation, the tips were annealed at $600^{\circ} \mathrm{C}$ for $1 \mathrm{~h}$ in the ultra-high vacuum chamber to obtain ordered $L 1_{0}$ structure.

The tip shapes were observed by scanning electron microscopy (SEM). In order to investigate the structural 

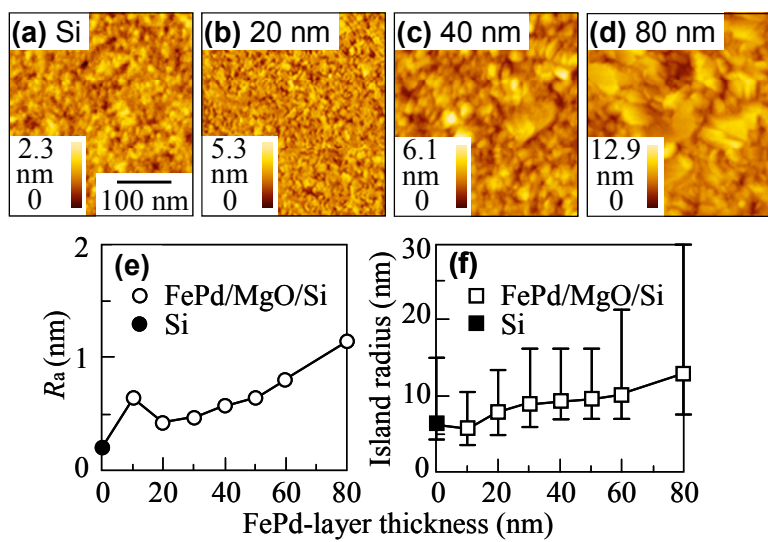

Fig. 1. (a)-(d) AFM images observed for (a) a Si substrate and (b)-(d) $\mathrm{FePd} / \mathrm{MgO}$ bi-layer films formed on $\mathrm{Si}$ substrates. The FePd-layer thicknesses are (b) 20, (c) 40, and (d) $80 \mathrm{~nm}$. (e, f) Dependences of FePd-layer thickness on (e) $R_{\mathrm{a}}$ and (f) island radius.

and the magnetic properties of coated films, $\mathrm{FePd} / \mathrm{MgO}$ bi-layer films were also deposited on flat $\mathrm{Si}$ substrates with natural oxide layers at RT and the samples were then annealed at $600{ }^{\circ} \mathrm{C}$ for $1 \mathrm{~h}$ under conditions similar to the case of MFM tip preparation. The arithmetical mean surface roughness $\left(R_{\mathrm{a}}\right)$ value of a $\mathrm{Si}$ substrate measured by atomic force microscopy (AFM) was $0.2 \mathrm{~nm}$. The film surface morphology was observed by AFM. The crystal structure was investigated by $\theta-2 \theta$ scan X-ray diffraction (XRD) with $\mathrm{Cu}-\mathrm{K} \alpha$ radiation $(\lambda=0.15418 \mathrm{~nm})$. The magnetic properties were measured by using a vibrating sample magnetometer.

MFM observation was carried out at RT under pressures lower than 0.1 Pa. MFM tips were magnetized along the tip axis so that the tip top possesses the south magnetic pole. A perpendicular medium recorded at linear densities from 1000 to 1700 kilo-flux-change-perinch $(\mathrm{kFCI})$ and a commercial HDD perpendicular medium with the areal density of $163 \mathrm{~Gb} / \mathrm{in}^{2}$ were used as observation samples. The quality factor value, the distance between tip and observation sample, and the scanning speed were respectively 3000-6000 (dimensionless quantity), $5-10 \mathrm{~nm}$, and $1.4 \mu \mathrm{m} / \mathrm{s}$.

\section{Results and discussion}

Figures 1(a) and (b)-(d) show the AFM images of a $\mathrm{Si}$ substrate and of $\mathrm{FePd} / \mathrm{MgO}$ bi-layer films with different FePd-layer thicknesses formed on Si substrates, respectively. Figures 1(e) and (f) show the thickness dependences on $R_{\mathrm{a}}$ and island radius, respectively. Here, the island radius is estimated by using the relation, $($ radius $)=[(\text { area }) / \pi]^{1 / 2}$. With increasing the thickness, the $R_{\mathrm{a}}$ and the island radius increase, suggesting that the radius of MFM tip increases. Figures 2(a-1)-(c-1) show the SEM images observed for a base-Si tip and of MFM tips coated with $\mathrm{FePd}(20 \mathrm{~nm}) / \mathrm{MgO}(5 \mathrm{~nm})$ and $\mathrm{FePd}(40$ $\mathrm{nm}) / \mathrm{MgO}(5 \mathrm{~nm})$ films. Island-like growth of $\mathrm{FePd} / \mathrm{MgO}$ bi-layer films on Si tips are recognized, similar to the case of film growth on flat Si substrates. Figures 2(a-2)-(c-2) show the high-magnification views of top parts of the tips shown in figures $2(\mathrm{a}-1)-(\mathrm{c}-1)$, respectively. The tip radius
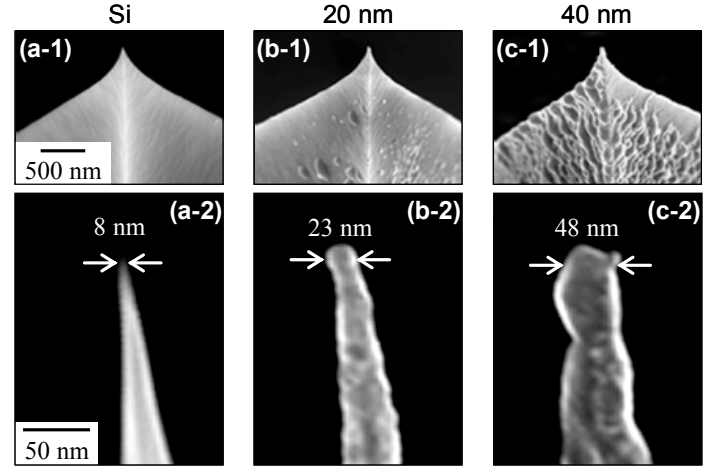

Fig. 2. SEM images observed for (a-1) a Si tip and (b-1, c-1) MFM tips coated with (b-1) FePd(20 nm)/MgO(5 nm) and (c-1) $\mathrm{FePd}(40 \mathrm{~nm}) / \mathrm{MgO}(5 \mathrm{~nm})$ bi-layer films. (a-2)-(c-2) Enlarged views of the areas around tip tops in (a-1)-(c-1), respectively.

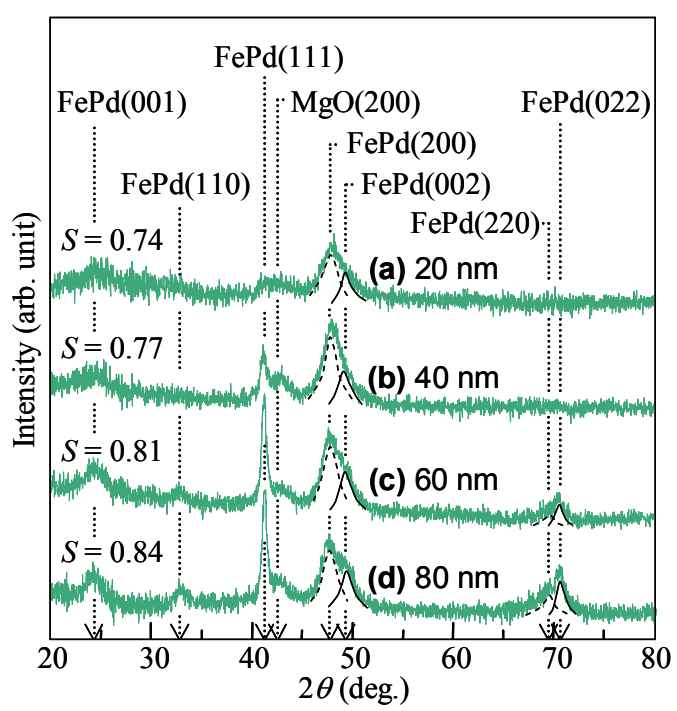

Fig. 3. XRD spectra of $\mathrm{FePd} / \mathrm{MgO}$ bi-layer films formed on $\mathrm{Si}$ substrates. The FePd-layer thicknesses are (a) 20, (b) 40, (c) 60, and (d) $80 \mathrm{~nm}$. The intensity is shown in a logarithmic scale.

increases with increasing the thickness, as expected from the AFM observations.

Figure 3 shows the XRD spectra of $\mathrm{FePd} / \mathrm{MgO}$ bilayer films formed on $\mathrm{Si}$ substrates. $\mathrm{FePd}(001)$ superlattice reflections are recognized in addition to $\mathrm{FePd}(002),(200)$, and (111) fundamental reflections for all the samples. FePd(110) superlattice and FePd(220) and (022) fundamental reflections are also observed when the FePd-layer thickness increases beyond $60 \mathrm{~nm}$. This is because the volumes of $\mathrm{FePd}(220)$ and (022) crystals are large enough to be detected by XRD for the FePd-layer thicknesses of 60 and $80 \mathrm{~nm}$. The order degrees of bilayer films with FePd-layer thicknesses of 20, 40, 60, and $80 \mathrm{~nm}$ are estimated from the $\mathrm{FePd}(001)$ and the $\mathrm{FePd}(002)$ reflections to be $0.74,0.77,0.81$, and 0.84 , respectively. The calculation method is reported in our previous paper [11]. The order degree is almost kept constant at around 0.8 for the FePd-layer thickness. $L 1_{0}$ ordered phase is also expected to be formed in the $\mathrm{FePd} / \mathrm{MgO}$ bi-layer films prepared on Si tips.

Figures 4(a)-(d) show the magnetization curves of $\mathrm{FePd} / \mathrm{MgO}$ bi-layer films formed on Si substrates. Figure 4(e) shows the thickness dependences on in-plane and 


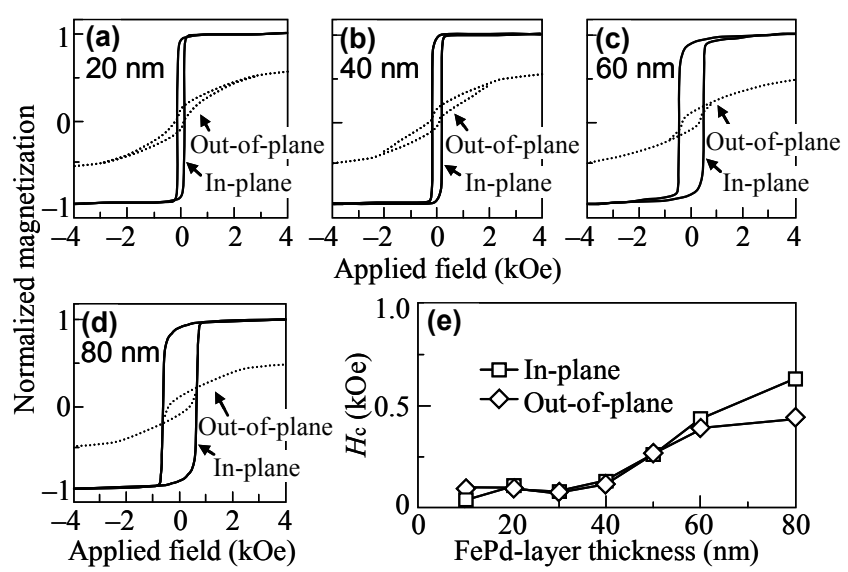

Fig. 4. (a)-(d) Magnetization curves measured for $\mathrm{FePd} / \mathrm{MgO}$ bi-layer films formed on $\mathrm{Si}$ substrates. The FePd-layer thicknesses are (a) 20, (b) 40, (c) 60, and (d) $80 \mathrm{~nm}$. (e) Dependences of FePd-layer thickness on in-plane and out-ofplane $H_{\mathrm{c}}$.

out-of-plane coercivities $\left(H_{\mathrm{c}}\right)$. As the thickness increases, the in-plane and the out-of-plane $H_{\mathrm{c}}$ increase. The result indicates that the $H_{\mathrm{sw}}$ of FePd-coated MFM tip increases with increasing the FePd-layer thickness.

Figures 5(a-1)-(g-1) show the MFM images of a same area of perpendicular medium recorded at $1000 \mathrm{kFCI}$ (bit length: $25.4 \mathrm{~nm}$ ) observed by using MFM tips coated with $\mathrm{FePd} / \mathrm{MgO}$ bi-layer films with different FePd-layer thicknesses. Figures 5(a-2)-(g-2) show the MFM signal profiles along the dotted lines in figures $5(\mathrm{a}-1)-(\mathrm{g}-1)$, respectively. Magnetic bit images corresponding to 1000 $\mathrm{kFCI}$ is observed for all the thicknesses. The MFM image observed by using the tip coated with 10 -nm-thick FePd layer is not clear, compared with the other images. This is possibly due to a low detection sensitivity related with decrease of (magnetization) $\times$ (coating thickness) value [9]. In order to investigate the resolution, MFM observations of magnetic bits recorded at linear densities ranging from 1000 to $1700 \mathrm{kFCI}$ were carried out. Figure 6(a) shows the MFM images of medium recorded at densities ranging from 1000 to $1700 \mathrm{kFCI}$ observed by using a tip coated with 20-nm-thick FePd film. Figures 6(b), (c), and (d), respectively, show the enlarged views of the areas surrounded by white square lines, the signal profiles along the dotted lines, and the power spectra analyzed for magnetic bit images in figure 6(a). Magnetic bits corresponding to densities ranging from 1000 to 1600 $\mathrm{kFCI}$ are clearly recognized. Thus, the MFM resolution is between $15.9 / 2=7.9 \mathrm{~nm}(1600 \mathrm{kFCI})$ and $14.9 / 2=7.5$ $\mathrm{nm}(1700 \mathrm{kFCI})$, that is, $7.7 \pm 0.2 \mathrm{~nm}$. Figure 7 summarizes the resolutions of tips with different FePdlayer thicknesses. As the thickness increases from 10 to $20 \mathrm{~nm}$, the resolution improves from 12.1 to $7.7 \mathrm{~nm}$, due to that the signal detection sensitivity is improved. With further increasing the thickness, the resolution gradually decreases. This is because the tip radius increases with increasing the thickness. The MFM resolution is apparently influenced by both the detection sensitivity and the tip radius.

For $H_{\mathrm{sw}}$ estimation, MFM observations of an HDD medium (163 Gb/in ${ }^{2}$ ) were performed for MFM tip repeatedly before and after applying a magnetic field. The

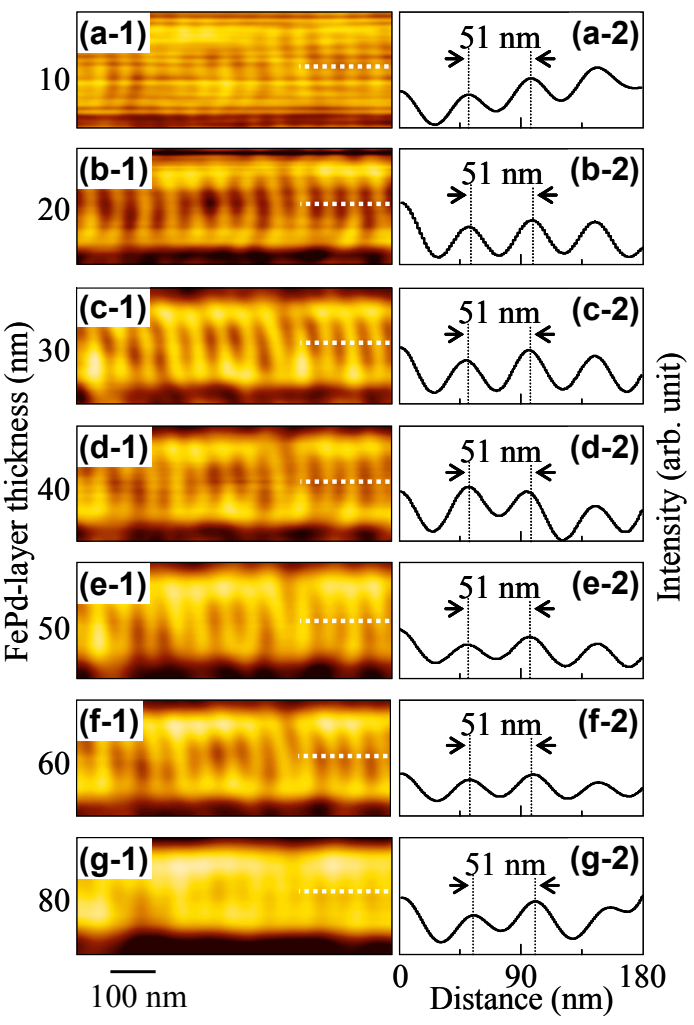

Fig. 5. (a-1)-(g-1) MFM images of a same area of perpendicular medium recorded at $1000 \mathrm{kFCI}$ observed by using MFM tips coated with $\mathrm{FePd} / \mathrm{MgO}$ bi-layer films. The FePd-layer thicknesses are (a) 10, (b) 20, (c) 30, (d) 40, (e) 50, (f) 60, and (g) $80 \mathrm{~nm}$. (a-2)-(g-2) MFM signal profiles along the dotted lines in (a-1)-(g-1), respectively.

magnetic field direction was opposite to the magnetization direction of MFM tip and the field was increased in a step-wise of $0.05 \mathrm{kOe}$. The $H_{\mathrm{sw}}$ was estimated as the magnetic field where the contrast of MFM image was reversed. Figures 8(a)-(c) show the MFM images of a same area of HDD medium observed by using a tip coated with 80 -nm-thick FePd film before and after applying magnetic fields. When a field of 1.45 $\mathrm{kOe}$ is applied for the tip [figure $8(\mathrm{~b})$ ], the MFM contrast does not change, when compared with the initial magnetization state shown in figure 8(a). With increasing the field up to $1.50 \mathrm{kOe}$, the contrast is reversed, as shown in figure $8(\mathrm{c})$. Thus, the $H_{\mathrm{sw}}$ is between 1.45 and $1.50 \mathrm{kOe}$ (i.e. $1.475 \pm 0.025 \mathrm{kOe})$. Figure $8(\mathrm{~d})$ shows the dependence of FePd-layer thickness on $H_{\text {sw }}$. With increasing the thickness from 10 to $80 \mathrm{~nm}$, the $H_{\mathrm{sw}}$ increases from 0.675 to $1.475 \mathrm{kOe}$. The $H_{\mathrm{sw}}$ of MFM tip coated with $\mathrm{FePd} / \mathrm{MgO}$ bi-layer film is larger than the $H_{\mathrm{c}}$ of $\mathrm{FePd} / \mathrm{MgO}$ bi-layer film deposited on flat Si substrate for respective thickness. The enhancement is possibly due to an influence of shape magnetic anisotropy. The magnetization state of MFM tip has been studied by using micromagnetic simulations [12-14]. It seems necessary to investigate the magnetization reverse process of FePdcoated tip by such theoretical calculations. The present study apparently shows that it is possible to prepare an MFM tip with resolution better than $10 \mathrm{~nm}$ and $H_{\mathrm{sw}}$ higher than $1 \mathrm{kOe}$ by coating a sharp Si tip with an $L 1_{0}$ ordered FePd film. 

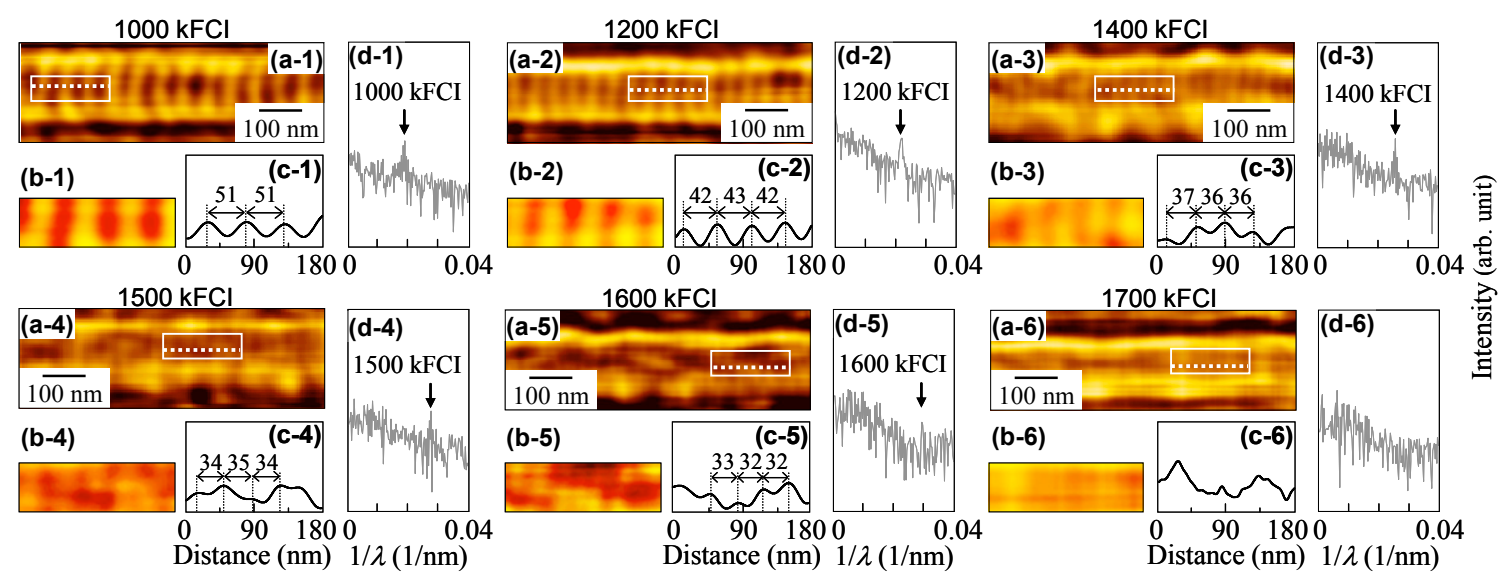

Fig. 6. (a-1)-(a-6) MFM images of a perpendicular medium recorded at (a-1) 1000, (a-2) 1200, (a-3) 1400, (a-4) 1500, (a-5) 1600, and (a-6) 1700 kFCI observed by using an MFM tip coated with 20-nm-thick FePd film. (b-1)-(b-6) Enlarged views of the areas surrounded by white square lines in (a-1)-(a-6), respectively. (c-1)-(c-6) Signal profiles along the dotted lines in (a-1)-(a-6), respectively. (d-1)-(d-6) Power spectra analyzed for the magnetic bit images of (a-1)-(a-6), respectively.

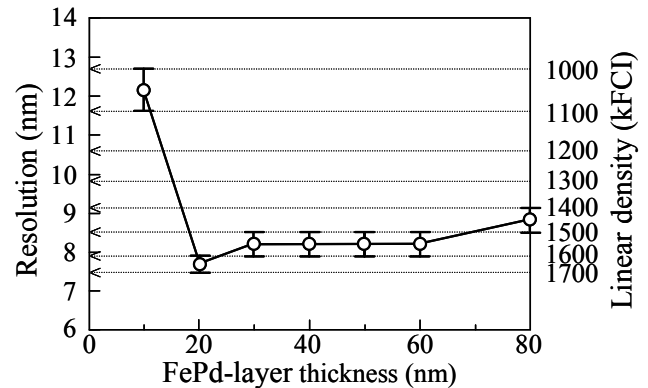

Fig. 7. Dependence of FePd-layer thickness on resolution.

\section{Conclusions}

MFM tips are prepared by coating Si tips with $L 1_{0}$ ordered FePd-alloy films. The effects of coating thickness on spatial resolution and switching field are investigated. With increasing the thickness from 10 to $20 \mathrm{~nm}$, the resolution improves from 12.7 to $7.9 \mathrm{~nm}$, due to improvement of signal detection sensitivity. As the thickness further increases, the resolution decreases. The reason is due to that the tip radius increases with increasing the thickness. The MFM resolution is affected by both the signal detection sensitivity and the tip radius. The switching field monotonically increases from 0.70 to $1.50 \mathrm{kOe}$ with increasing the coating thickness from 10 to $80 \mathrm{~nm}$. An FePd-coated MFM tip is useful to observe the magnetization structures of future high-density HDD media.

\section{Acknowledgments}

Authors thank Ms. Noriko Saidoh of RIMCOF for SEM observations. A part of this work was supported by METI and JSPS (Grant-in-Aid for Scientific Research, No. (C) 22560302), Japan.

\section{References}

1. A. J. den Boef, Appl. Phys. Lett. 56, 2045 (1990)

2. M. Rührig, S. Porthun, J. C. Lodder, Rev. Sci. Instrum. 65, 3224 (1994)
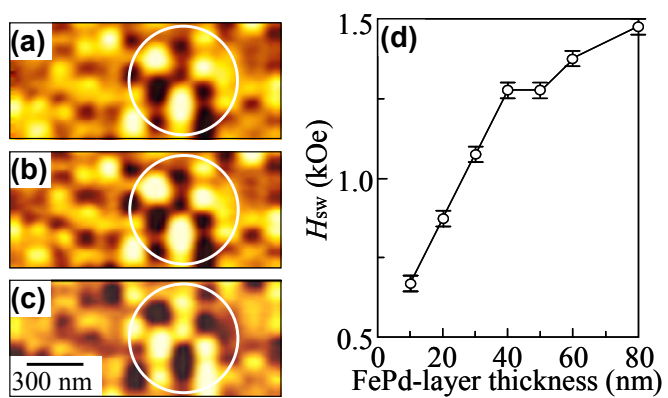

Fig. 8. MFM images of a same area of HDD perpendicular medium observed by using an MFM tip coated with 80-nmthick FePd film (a) before and (b, c) after applying magnetic fields of (b) 1.45 and (c) $1.50 \mathrm{kOe}$. The contrast is reversed for (c). (d) Dependence of FePd-layer thickness on $H_{\mathrm{sw}}$.

3. Z. Liu, Y. Dan, Q. Jinjun, Y. Wu, J. Appl. Phys. 91, 8843 (2002)

4. G. N. Phillips, M. Siekman, L. Abelmann, J. C. Lodder, Appl. Phys. Lett. 81, 865 (2002)

5. I. Utke, P. Hoffmann, R. Berger, L. Scandella, Appl. Phys. Lett. 80, 4792 (2002)

6. L. Gao, L. P. Yue, T. Yokota, R. Skomski, S. H. Liou, H. Saito, S. Ishio, IEEE Trans. Magn. 40, 2194 (2004)

7. N. Amos, R. Ikkawai, R. Haddon, D. Litvinov, S. Khizroev, Appl. Phys. Lett. 93, 3116 (2008)

8. K. Nagano, K. Tobari, M. Ohtake, M. Futamoto, J. Phys.: Conf. Ser. 303, 012014 (2011)

9. K. Nagano, K, Tobari, K. Soneta, M. Ohtake, M. Futamoto, J. Magn. Soc. Jpn. 36, 109 (2012)

10. M. Ohtake, K. Soneta, M. Futamoto, J. Appl. Phys. 111, 07E339 (2012)

11. M. Ohtake, S. Ouchi, F. Kirino, M. Futamoto, J. Appl. Phys. 111, 07A708 (2012)

12. V. Cambel, P. Eliáš, D. Gregušová, J. Martaus, J. Fedor, G. Karapetrov, V. Novosad, J. Magn. Magn. Mater. 322, 2715 (2010)

13. T. Manago, H. Asada, T. Uzumaki, F. Takano, H. Akinaga, H. Kuramochi, Nanotechnology 23, 035501 (2012)

14. Hongjia Li, Dan Wei, N. Piramanayagam, J. Appl. Phys. 111, 07E309 (2012) 